\author{
Jorge A. Combi, Juan F. Albacete Colombo, Gustavo E. Romero, Paula \\ Benaglia
}

\title{
Hard X-ray emission from the SNR G337.2+0.1
}

Received: date / Accepted: date

\begin{abstract}
We report the hard X-ray emission of the SNR candidate G337.2+0.1. The object shows centrally filled and diffuse X-ray emission, which peaks in the hard 3.0-10.0 keV band. A spectral study confirms that the column density of the central part of the object is about $\mathrm{N}_{\mathrm{H}} \sim 5.9( \pm 1.5) \times 10^{22} \mathrm{~cm}^{-2}$ and its X-ray spectrum is well represented by a single power-law with a photon index $\Gamma=0.96 \pm 0.56$. Detailed spectral analysis indicates that the outer region is highly absorbed and quite softer than the inner region. Such characteristics are already observed in other X-ray plerions. Based on the morphological and spectral X-ray information, we confirm the SNR nature of G337.2+0.1, and suggest that the central region of the source is a pulsar wind nebula (PWN), originated by an energetic though yet undetected pulsar, that is currently losing energy at a rate of $\sim 10^{36} \mathrm{erg} \mathrm{s}^{-1}$.
\end{abstract}

Keywords X-rays: individual (AX J1635.9-4719)

- (ISM:) supernova remnants — ISM: individual

(G337.2+0.1) - X-rays: ISM — radiation mechanisms: non-thermal

PACS First $\cdot$ Second $\cdot$ More

\section{Introduction}

Supernova remnants (SNRs) of the Crab-like or plerionic class are objects characterized in the radio band by a compact, filled-center morphology with a relatively flat

Jorge A. Combi

Departamento de Física (EPS), Universidad de Jaén, Campus Las Lagunillas s/n, 23071 Jaén, Spain

Tel.: +34-953-212473

Fax: +123-45-678910

E-mail: jcombi@ujaen.es

Juan F. Albacete Colombo

Osservatorio Astronomico di Palermo, Piazza del Parlamento

1, Palermo (90141), Italy

Gustavo E. Romero, Paula Benaglia

Instituto Argentino de Radioastronomía, C.C.5, (1894) Villa

Elisa, Buenos Aires, Argentina spectral index [19]. In the X-ray band they present nonthermal spectra, characteristic of synchrotron emission. The non-thermal spectrum, in some cases, can even reach the very high-energy gamma-ray region, like in MSH 1552, G18.0-07, and Vela X $[1,2]$. It is widely believed that plerions are powered by the loss of rotational energy from energetic pulsars, although clear evidence of the presence of these objects is often lacking. The pulsar wind forms a nebula inside the SNR (the PWN), where relativistic particles can be efficiently accelerated producing synchrotron radiation that yields the typical morphologies observed at radio and X-rays.

Recently, [11] have presented evidence supporting a SNR origin for the radio source G337.2+0.1. A thorough study of its radio (continuum and line) and X-ray properties shows that the emission from the source is consistent with what is expected for a young SNR located at a distance $d \sim 14 \mathrm{kpc}$ [11]. More recently, G337.2+0.1 has been suggested as the potential counterpart of the high-energy gamma-ray source HESS J1634-472 [3]: this possibility needs further confirmation. Throughout this paper, we adopt $14 \mathrm{kpc}$ as the distance to G337.2+0.1 (hence, 1' corresponds to $4 \mathrm{pc}$ ).

In this paper, we present the first XMM observations of the SNR candidate G337.2+0.1. Based on its X-ray properties we are able to confirm that this object is a non-thermal SNR with hard, featureless power-law spectrum and, possibly, a PWN originated by a non-detected energetic pulsar.

\section{X-ray Observations}

The SNR candidate G337.2+0.1 has been marginally observed on February 2004 by the XMM X-ray satellite in two separated observations (Obs-Id. 0204500201 and 0204500301). Both observations were acquired with the EPIC MOS [24] and EPIC PN [21] cameras.

Unfortunately, the first observation was affected by a high and variable soft proton background level [13], whereas the second one (Id. 0204500301) is unaffected 


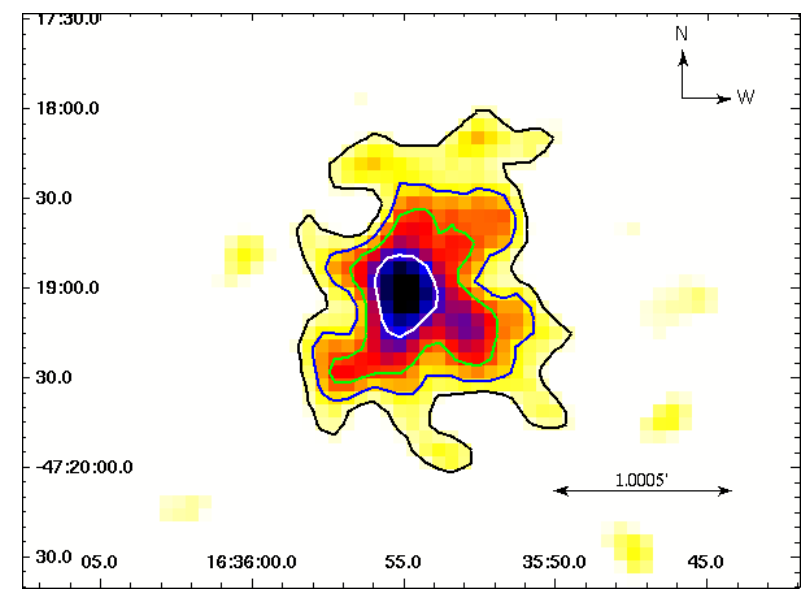

Fig. 1 X-ray images of the G337.2+0.1 in the $0.5-10.0 \mathrm{keV}$ band. Typical local background level of the smoothed image is $\sim 0.94( \pm 0.17)$ counts $\mathrm{px}^{-1}$. Contours show the level of 1.6 $(\sim 1 \sigma), 3.2(\sim 2 \sigma), 4.7(\sim 3 \sigma)$ and $6.3(\sim 4 \sigma)$ photons $\mathrm{px}^{-1}$, from outer to inner curves, respectively.

by background fluctuations. We derive Good Time Intervals (GTI) by the accumulation of background lightcurves in the 10-15 keV energy band, which leads to a reduction of $\sim 87 \%$ in the net exposure time of the Obs-Id. 0204500201. In order to avoid contamination for high background patterns hereafter our analysis concerns only to the observation 0204500301. The number of detected counts in the 0.5-2.5 and 2.5-10.0 keV energy bands are $117 / 121 / 315$ and $431 / 403 / 1154$ for the MOS1, MOS2 and PN cameras, respectively. Finally, at the SNR G337.2+0.1 EPIC-PN position there is a CCD gap in the X-ray image, leading us to ignore this data only for the X-ray image analysis section, but they are included for the rest of our study.

\section{$3 \mathrm{X}$-ray analysis of G337.2+0.1}

The coordinates of the SNR G337.2+0.1 were defined at the position where X-ray emission peaks $\left(\alpha_{\mathrm{J} 2000.0}=16^{\mathrm{h}}\right.$ $\left.35^{\mathrm{m}} 54^{\prime} .95, \delta_{\mathrm{J} 2000.0}=-47^{\circ} 19^{\prime} 02 " 2\right)$. This position agrees well with the previous estimate of the radio position [11], but differs in $\sim 50$ arcsec from the ASCA coordinates.

Figure 1 shows the X-ray image of the SNR G337.2+0.1 in the $0.5-10.0 \mathrm{keV}$ energy band. The image does not reveal a typical rim-brightened outer SNR shell, so the overall size of the diffuse X-ray emission is uncertain.

Plotted contours corresponds to 1, 2, 3 and 4 sigma levels over the mean background flux of the image $\left(\sim 6 \times 10^{-}\right.$ $\left.\mathrm{ph} / \mathrm{cm}^{2} / \mathrm{s}\right)$.

Finally, we are able to investigate the spatial extent of G337.2+0.1. According to the image presented in Figure 1, G337.2+0.1 does not extend farther than 1.5 arcmin from the central peak. We also compare G337.2+0.1 spatial extent with that produced by a point source placed at a similar off-axis ( $\sim 6$ arcmin $)$ position. In Figure 2 we

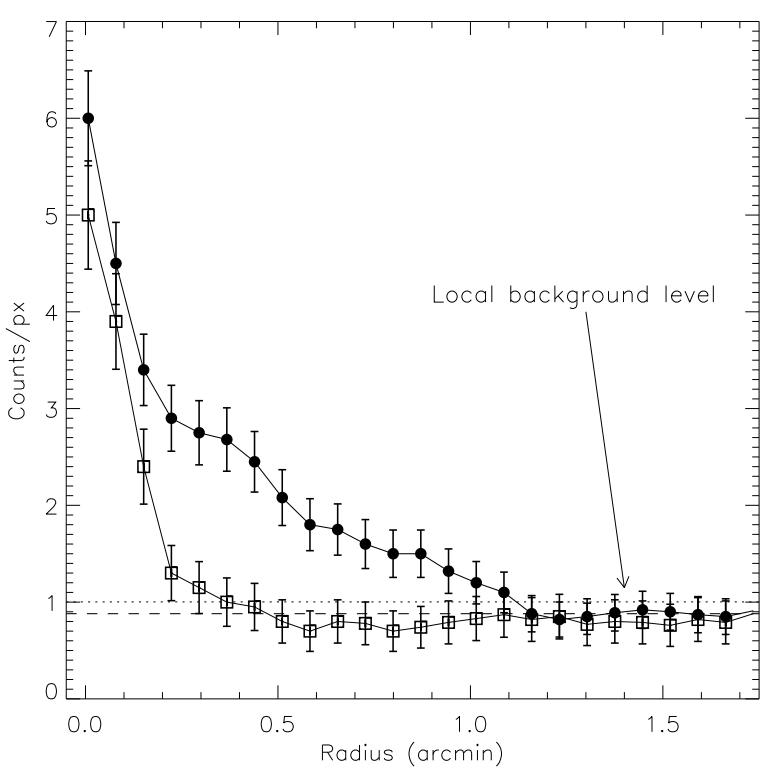

Fig. 2 Radial profile of the smoothed X-ray image of SNR G337.2+0.1 (filled circle). Open squares refers to the radial profile of an observed for a point source located at roughly the same off-axis position

show that the SNR G337.2+0.1 has an extension $~ 3.5$ times larger than what is expected for a point-like source.

\subsection{Spectral analysis}

For the spectral analysis we used MOS and PN data. It was performed using the XSPEC package [5]. Since the statistics of the source is not complete enough to perform a spatial-spectral analysis, we extracted X-ray photon events from only three different regions: $i$ ) a circular region of 50 arcsec; $i$ ) a circular region of only 12 arcsec that accounts for the central source observed in the image; $i i i)$ an annulus for the extended emission of innerouter radii of $12-50$ arcsec. The background region was taken from a nearby blank region in the neighborhood of the source. The background-subtracted spectra of the MOS and PN data (the upper line) are shown in Figure 3.

Our analysis of the XMM, EPIC spectra was essentially performed using a single non-thermal model, described by a simple Power-Law emission model. We also fit the spectra by a thermal emission model [9], but parameters of this model are suitable to discuss in terms of -5rigin of a thermal X-ray emission.

According to the results obtained, central part of the SNR appears quite harder $(\Gamma \sim 0.96)$ than the outer one $(\Gamma \sim 2.38)$. We suggests that the most reasonable interpretation of observed emission from central part of SNR is synchrotron radiation from relativistic electrons accelerated in the vicinity of the central source of the SNR. The softening of the spectrum toward the outer 

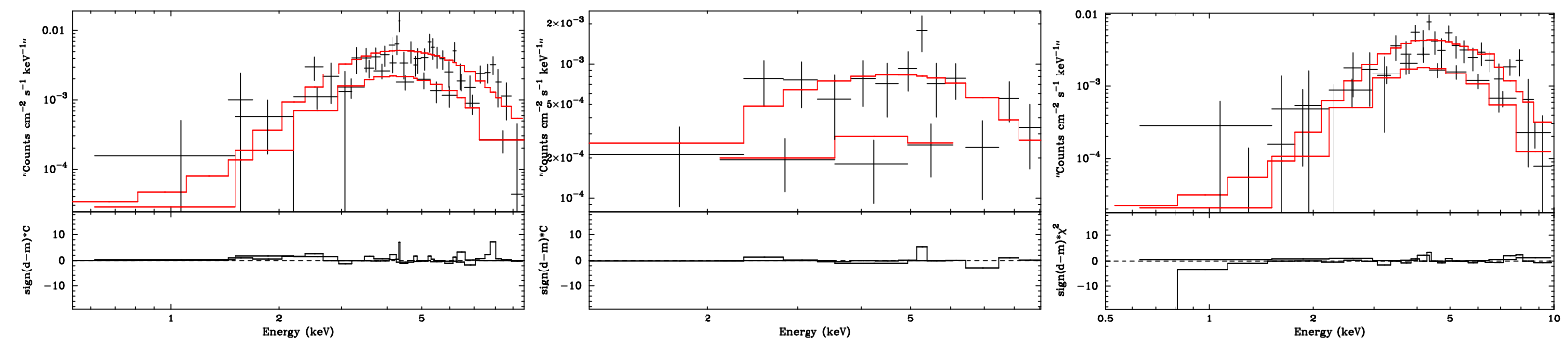

Fig. 3 X-ray spectra of the SNR G337.2+0.1 in the 0.5-10.0 keV band. In all figures PN data are the upper line. Left: Spectrum extracted on the whole SNR. Center: Spectrum of the compact source observed in the center of SNR G337.2+0.1. Right: Outer spectrum that excludes the central contribution.

regions of the nebula is a well known effect which has been seen in other X-ray plerions (e.g., G0.9+0.1, [15]; 3C58, [23]; G21.5-0.9, [19]).

To get a statistical assessment of the X-ray variability of the SNR G337.2+0.1, we use the 32.6-ksec EPIC$\mathrm{PN}$ observation to compare the time arrival distribution of source photons by means of the Kolmogorov-Smirnov (KS) test [17]. According to the Nyquist limit, no significant periodic signal can be seen with a period larger than twice the read-out time of the EPIC-PN camera in the FF mode. This means a limit of $2 \times 200 \mathrm{~ms}=400 \mathrm{~ms}$.

\section{Discussion}

The X-ray morphology of G337.2+0.1 shows a centrally peaked emission, surrounded by a diffuse X-ray nebula. The lightcurve of the object does not show any significant flux variability above $0.4 \mathrm{seg}$, implying that at first glance, a pulsar origin for the central contribution could be ruled out. However, a detailed spectral analysis indicates that the outer region is softer than the inner region, a phenomenon observed previously in several X-ray plerions with PWN (e.g., G0.9+0.1, [15]; 3C58, [23]).

A spectral analysis of the central component of the SNR show that the X-ray spectrum is well represented by a single power-law with a photon index $\Gamma=0.96 \pm 0.56$, a value similar to that of objects powered by an energetic pulsar [12]. Moreover, the non-detection of line emission in this spectrum is consistent with synchrotron radiation from a population of relativistic electrons. These facts suggest a non-thermal origin for the X-ray emission. We therefore conclude that the system G337.2+0.1/ AX J1635.9-4719 is a non-thermal SNR with, possibly, a non-detected pulsar.

Possible reasons for the non-detection of a pulsar inside the SNR are a short rotation period (less than 400 $\mathrm{ms}$ ) or unfavorable geometrical conditions. The presence of a pulsar is suggested by the central X-ray peak found inside G337.2+0.1. In what follows we explore the possibility that there exists a hidden pulsar-powered component (plerion) within the SNR. Using the empirical formula derived by [18], $\log L_{\mathrm{X}}\left(\mathrm{erg} \mathrm{s}^{-1}\right)=1.39 \log \dot{E}-16.6$, where $L_{\mathrm{X}}$ is the $\mathrm{X}$-ray luminosity of the plerion in the
0.2-4 keV band, we can make an estimate of spin-down luminosity of the pulsar (see also, [6]). Using the Xray flux of the compact source and its nebula, $F_{\mathrm{X}}(0.2-4$ $\mathrm{keV})=4.9 \pm 1.7 \times 10^{-13} \mathrm{erg} \mathrm{s}^{-1} \mathrm{~cm}^{-2}$, we get $L_{\mathrm{X}}=1.1 \times 10^{34}$ $\mathrm{erg} \mathrm{s}^{-1}$ (unabsorbed). This implies a spin-down luminosity of $\dot{E} \sim 2.5 \times 10^{36} \mathrm{erg} \mathrm{s}^{-1}$, and a period of $P \geq 0.08$ $\left(t_{3} \dot{E}_{38}\right)^{1 / 2}(\mathrm{~s})$, where $\dot{E_{38}}$ is the spin-down luminosity in units of $10^{38} \mathrm{erg} \mathrm{s}^{-1}$, and $t_{3}$ is the pulsar age in units of $10^{3}$ years. In order to compare this result with others empirical relations between the X-ray luminosity and the rate of the spin-down energy loss, we have used the [6] and [16] equations. In the first case, (taking into account only the X-ray flux of the point source in the $0.1-2.4 \mathrm{keV}$ band) the spin-down luminosity is $\dot{E} \sim 3 \times 10^{35} \mathrm{erg} \mathrm{s}^{-1}$, a factor 9 lower than the value obtained with the [18] relation. In the second case (using the X-ray flux of the compact source and its nebula in the $2-10 \mathrm{keV}$ band), $\dot{E} \sim 8 \times 10^{36} \mathrm{erg} \mathrm{s}^{-1}$, this value is a factor 3 higher than the value obtained with the [18] relation. If we assume a pulsar period of less than $0.4 \mathrm{~s}$, we obtain an upper limit for the age of the pulsar of $t \leq 1000$ years.

We have seen that G337.2+0.1 does not show a rimbrightened outer SNR shell. This could be the result of the absorption of the soft thermal emission from the forward shock by the very high absorbing column density. Other sources like Crab, G21.5-0.9 [19] and 3C58 [23] have weak or absent X-ray rims and all are powered by young X-ray pulsars ([14]; [10]).

It could be interesting to compare the characteristics of G337.2+0.1 with 3C58. The X-ray luminosities, between 0.5 and $10.0 \mathrm{keV}$, are $\sim 4.8 \times 10^{34} \mathrm{erg} \mathrm{s}^{-1}$ and $\sim 2.4 \times 10^{34} \mathrm{erg} \mathrm{s}^{-1}$, respectively. The radio luminosities, at $1 \mathrm{GHz}$, are $\sim 3 \times 10^{32} \mathrm{erg} \mathrm{s}^{-1}$ and $\sim 4 \times 10^{32} \mathrm{erg}$ $\mathrm{s}^{-1}$. We see, then, that both sources are quite similar. We notice that the estimated age of $3 \mathrm{C} 58$ is $\sim 800 \mathrm{yr}$. The most significant difference seems to be the absence of any thermal component in the case of G337.2+0.1. If we compare with the Crab, on the contrary, we see that the Crab pulsar is injecting around two orders of magnitude more energy per time unit in the nebula than G337.2+0.1. The spin-down luminosity inferred for the pulsar from the new X-ray data sets an upper limit to the energy available for high-energy cooling channels like inverse Compton scattering and proton-proton interac- 
tions. The luminosity of the nearby HESS source J1634$472(E>1 \mathrm{TeV})$, if it is located at the same distance inferred for G337.2+0.1, would be $L_{\gamma} \sim 7 \times 10^{34} \mathrm{erg} \mathrm{s}^{-1}$. So, a physical association would be possible only if $\sim 7 \%$ of the spin-down luminosity is converted in high-energy $\gamma$-rays.

Complementary studies of the PWN scenario will involve high-resolution X-ray observations with Chandra satellite, and radio observations with ATCA, to allow the comparison of the X-ray spectrum and morphology with those at the radio band. GLAST observations could reveal a $\mathrm{GeV} \gamma$-ray source if the proposed association with HESS J1634-472 is correct.

\section{References}

1. Aharonian, F., et al. 2005, Astron. Astrophys., 442, L25.

2. Aharonian, F., et al. 2006a, Astron. Astrophys., 448, L43.

3. Aharonian, F., et al. 2006b, Astrophys. J., 636, 777.

4. Anders, E., \& Grevesse, N. 1989, Geochimica et Cosmochimica Acta, 53, 197.

5. Arnaud, K. A. 1996, ASP Conf. Ser. 101: Astronomical Data Analysis Software and Systems V, 101, 17.

6. Becker, W., \& Truemper, J. 1997, Astron. Astrophys., 326, 682

7. Bietenholz, M. F., Kassim, N. E., \& Weiler, K. W. 2001, ApJ, 560, 772

8. Bocchino, F., \& Bykov, A. M. 2001, Astron. Astrophys., $376,248$.

9. Brickhouse, N. S. 2003, IAUJD, 17, 23.

10. Camilo, F., et al. 2006, Astrophys. J., 637, 456

11. Combi, J.A., Benaglia, P., Romero, G.E., Sugizaki, M., 2005, A\&A, 431, L9.

12. Gotthelf, E. V. 2003, Astrophys. J., 591, 361

13. Lumb, D. H., Warwick, R. S., Page, M., \& De Luca, A. 2002, Astron. Astrophys., 389, 93.

14. Murray, S. S., et al. 2002, Astrophys. J., 568, 226

15. Porquet, D., Decourchelle, A., \& Warwick, R. S. 2003, Astron. Astrophys., 401, 197 .

16. Possenti, A., Cerutti, R., Colpi, M., Mereghetti, S. 2002, Astron. Astrophys., 387, 993

17. Press, W. H., Teukolsky, S. A., Vetterling, W. T., \& Flannery, B. P. 1992, Cambridge: University Press, — c1992, 2nd ed.

18. Seward, F. D., \& Wang, Z.-R. 1988, Astrophys. J., 332, 199.

19. Slane, P., Chen, Y., Schulz, N. S., et al. 2000, ApJL, 533, L29.

20. Stephenson, F. R., \& Green, D. A. 2002, Historical Supernovae and their Remnants (Oxford: OUP)

21. Strüder, L., et al. 2001, Astron. Astrophys., 365, L18.

22. Sugizaki, M., et al. 2001, ApJS, 134, 77.

23. Torii, K., Slane, P. O., Kinugasa, K., Hashimotodani, K., \& Tsunemi, H. 2000, PASJ, 52, 875 .

24. Turner, M. J. L., et al. 2001, Astron. Astrophys., 365, L27.

25. Yamaguchi, H., Ueno, M., Koyama, K., et al. 2004, PASJ, 56, 1059 